\title{
Transverse Sectioning of Mature Rice (Oryza sativa L.) Kernels for Scanning Electron Microscopy Imaging Using Pipette Tips as Immobilization Support
}

\author{
Jordan Demone ${ }^{1}$, Kiah A. Barton ${ }^{1,2}$, Illimar Altosaar ${ }^{1,2}$ \\ ${ }^{1}$ Department of Biochemistry, Microbiology, and Immunology, University of Ottawa ${ }^{2}$ Proteins Easy Corp
}

\section{Corresponding Author}

Illimar Altosaar

altosaar@uottawa.ca

\section{Citation}

Demone, J., Barton, K.A.,

Altosaar, I. Transverse Sectioning of

Mature Rice (Oryza sativa L.) Kernels for

Scanning Electron Microscopy Imaging

Using Pipette Tips as Immobilization

Support. J. Vis. Exp. (179), e61407,

doi:10.3791/61407 (2022).

\section{Date Published}

January 25, 2022

DOI

$10.3791 / 61407$

URL

jove.com/video/61407

\section{Abstract}

Starch granules (SGs) exhibit different morphologies depending on the plant species, especially in the endosperm of the Poaceae family. Endosperm phenotyping can be used to classify genotypes based on SG morphotype using scanning electron microscopic (SEM) analysis. SGs can be visualized using SEM by slicing through the kernel (pericarp, aleurone layers, and endosperm) and exposing the organellar contents. Current methods require the rice kernel to be embedded in plastic resin and sectioned using a microtome or embedded in a truncated pipette tip and sectioned by hand using a razor blade. The former method requires specialized equipment and is time-consuming, while the latter introduces a new host of problems depending on rice genotype. Chalky rice varieties, particularly, pose a problem for this type of sectioning due to the friable nature of their endosperm tissue. Presented here is a technique for preparing translucent and chalky rice kernel sections for microscopy, requiring only pipette tips and a scalpel blade. Preparing the sections within the confines of a pipette tip prevents rice kernel endosperm from shattering (for translucent or 'vitreous' phenotypes) and crumbling (for chalky phenotypes). Using this technique, endosperm cell patterning and the structure of intact SGs can be observed.

\section{Introduction}

Starch granules (SGs) exhibit different morphologies depending on the plant species, especially in the endosperm of the Poaceae family ${ }^{1,2}$. Endosperm phenotyping can be used to classify genotypes based on SG phenotype using scanning electron microscopic analysis. SGs can be visualized using scanning electron microscopy (SEM) by slicing the kernel and prying away the endosperm cell walls ${ }^{2}$.

The purpose of this technique is to easily prepare transverse rice kernel sections solely for the rapid SEM analysis. The development of this technique was motivated by the necessity for a rapid cross sectioning approach whereby 
samples are prepared for SEM microscopy immediately before visualization using minimal equipment.

This technique involves the insertion of the husked rice kernel into the pipette tip for complete immobilization. This is particularly important when cross-sectioning chalky rice kernel phenotypes, which are friable and easily crumble under pressure $^{3}$. Chalkiness is an undesirable quality in rice since it affects the appearance of the kernel and causes the kernel to break easily during polishing and milling ${ }^{3}$. Chalkiness presents as an opaque area in a cross-section of the kernel that can be observed by the naked eye; at the microscopic level, chalkiness is characterized by small, loosely packed starch granules. Causes of chalkiness can be genetic ${ }^{4,5}$ or environmental $^{6,7}$.

Cereal seed cross sections have traditionally been prepared using chemical fixing methods and sectioning following sample embedding into paraffin wax or another solid matrix $4,8,9,10$. In 2010, the Matsushima method was introduced as a way to avoid complicated and timeconsuming rice kernel sample preparation ${ }^{4}$. This method involved the insertion of the husked rice kernel into a truncated pipette tip. The tip is held stationary by a block trimmer, and thin, partial endosperm sections are harvested using a hand-held razor blade. Another rapid technique developed in 2016 allowed for thin whole sectioning of a wide variety of dry seeds, including chalky varieties ${ }^{10}$. These methods motivated the development of the rapid technique presented here.

This new technique is appropriate for researchers who want to obtain intact transverse cross sections of rice kernels for endosperm phenotyping and starch morphology analysis using SEM.
This protocol represents an adaptation of the Matsushima truncated pipette tip method $^{4}$, with several notable modifications: (1) kernels are not imbibed at any point of the technique; (2) neither a block trimmer nor an ultramicrotome are required to prepare the sections. A wild type 'translucent' cultivar (Oryza sativa L. ssp. japonica cv. Nipponbare) and a mutagenized 'chalky' line of Nipponbare (ssg1, substandard starch grain 1$)^{4}$ were examined in this study. These two cultivars were selected for the analysis here to demonstrate the technical and visual differences in processing translucentand chalky-type rice sections.

\section{Protocol}

\section{Preparation of transverse rice section}

1. De-husk dry, intact kernels as shown in Figure 1A.

1. Loosen the husks and dehull rice kernels by grinding the kernels between two flat rubber stoppers. Remove the husked rice kernels from the panicle, if necessary.

2. Place a single kernel onto a flat rubber stopper on a work bench (Figure 1B). Ensure that this stopper remains stationary.

3. Use a second flat rubber stopper (Figure 1C) to abrade the kernel by twisting it in against the first rubber stopper, using sufficient pressure (Figure 1D). Remove husks from the kernel, taking care not to shatter the endosperm. Remove any remaining husk using fine forceps. A de-husked kernel is shown in Figure 1E.

2. Using fine forceps, insert an individual husked kernel into a plastic pipette tip (250 $\mu \mathrm{L}$ size, one seed/tip) (Figure 
1F). Ensure that the embryo end of the kernel is facing towards the (conical) end of the pipette tip (Figure 1G). NOTE: Inserting the kernel this way ensures that the kernel will fit as snugly as possible into the pipette tip as the kernel is narrower towards its proximal end.

3. Insert a second $250 \mu \mathrm{L}$ pipette tip to force the kernel into the pipette tip and to keep the kernel immobile during sectioning, taking care not to damage the kernel or bend the second pipette tip (Figure 1H). The proper 'telescope' assemblage is indicated in Figure 11.

4. Lay the pipette tip assemblage flat on a work bench and hold in place by hand (Figure 1J). With the other hand, use a sharp scalpel blade (No. 20) to slice through the center of the kernel and cut the end of the pipette tip off (Figure 1K). Using the scalpel cut $1 \mathrm{~mm}$ thick sections of the rice kernel (Figure 1L).

NOTE: The kernel section is tightly enclosed within an annulus of plastic (Figure 1M). Mean section thickness for three exemplar genotypes are found in Table 1. Sections significantly thinner than $1 \mathrm{~mm}$ will shatter or crumble. It is important to note from what part of the kernel each section originates if this experiment is being performed on several varieties of rice for comparison, as starch morphology does vary throughout the endosperm ${ }^{11}$.

\section{Reflected light microscopy of transverse rice sections}

1. Use fine forceps to place the transverse rice sections (prepared in section 1) on a black piece of heavy gauge black paper.

2. Obtain light images of transverse sections of Nipponbare using a stereomicroscope with mounted goosenecks for oblique illumination, as shown in Figure 1N-S.
3. Observe endosperm morphology under at least $10 x$ magnification.

NOTE: Any epilight source is preferable to bright field microscopy as sections obtained using this technique are not thin enough for the light to pass through.

\section{Scanning electron microscopy of transverse rice sections}

1. Place the samples onto a carbon disk stuck to an aluminum stub and place onto a charge reduction sample holder. Remove the plastic ring from the pipette tip mount using fine forceps to prevent the plastic from entering the vacuum apparatus of the SEM.

NOTE: Images of endosperm cells, SGs, and subgranules are obtained using a desktop SEM machine that does not require samples to be sputter coated.

2. Obtain the images using a high sensitivity multi-mode backscatter electron (BSE) detector at $10 \mathrm{kV}$.

\section{Representative Results}

Wild type Nipponbare (Figure 2A) and ssg1 sections (Figure 2B) were examined under three magnifications: 260x, 920x, and 4200x. This technique allows for the preparation of sections of sufficient quality to observe the entire endosperm cell (Figure 3A), compound starch granules (Figure 3B), and individual subgranules (Figure $3 \mathbf{C}$ ). Husked kernels take longer to process than polished kernels as the dry hulls need to be removed by abrasion before sectioning. Chalky kernels also take longer to process than polished translucent kernels, as care must be taken to not shatter the kernel during sectioning. A properly prepared rice section should be approximately $0.9 \mathrm{~mm}$ thick (Table 1 ) with minimal to no shattering of the endosperm (Figure $1 \mathbf{N}$ ) and intact pericarp and aleurone layers (Figure 10). Improper placement of the 
scalpel on the pipette tip when sectioning can lead to 'chipped' sections (Figure 1P). Similarly, bright field images of optimal transverse sections of ssg1 (Figure 1Q) demonstrated intact endosperm, pericarp, and aleurone layers intact and available for visualization (Figure 1R). A broken chalky kernel section (Figure 1S) may still be useable for visualization if the only purpose is to observe SGs, but endosperm cell pattern will not be visible. A broken section may be difficult to handle for analysis. More shearing of endosperm cell walls was observed in wild type Nipponbare, as the cells are more tightly packed and less friable than the ssg1 kernels. No shearing of endosperm cells was observed in the ssg1 sections and compound starch granules are intact.

Figure $\mathbf{S 1}$ demonstrates the reliability of results using the 'telescope' technique to section rice kernels. Rice lines identified as translucent kernel producers - wild type Resistant Starch (RS) hybrid line Xieyou 7954 (Oryza sativa L. ssp. indica) ${ }^{12,13,14}$ (Figure S1A) and cobalt-generated mutant RS111 13,15 (Figure S1B) produced sections through which light was visible using a stereomicroscope. The corresponding SEM images revealed that these lines produce the 'normal' rice endosperm phenotype: tightly packed, polyhedral starch granules. Chalky kernel producers, commercial variety Yi-Tang ${ }^{16}$ (Figure S1C) and $\mathrm{RS}^{13}$, a mutant of RS111 15 (Figure S1D), exhibited white, opaque kernel sections. The corresponding SEM images displayed markedly different morphology compared to the wild type translucent RS background line: starch granules were round and loosely packed. Wild type Xiushui 11 (Oryza sativa L. ssp. japonica) (Figure S1E) and its mutant, KMD1 (Kemingdao1), which express the Cry1Ab gene to inhibit insect predation $^{17,18,19}$ (Figure S1F) exhibited sections and endosperm morphotypes similar to the translucent RS lines.

The technique presented here is optimal for preparing samples of chalky-type rice kernels for phenotypic analysis, but also provides advantages for sectioning translucent rice kernel phenotypes ${ }^{20}$ : slicing the samples using pressure from above reduces risk of shattering of the endosperm and dislocation. Samples can easily be prepared within seconds (Table 2). Multiple genotypes were analyzed using this technique to test its efficacy (Table 3). As shown in Figure S2, this technique can be applied to seeds of other species. The model monocot Brachypodium distachyon produces very hard seeds containing only B-granule starch ${ }^{21}$, which lack puroindoline $\mathrm{A}$, a protein that confers softness to starch granules $^{22}$. It was still possible to obtain an intact transverse section (Figure S2A). Obtaining an intact transverse section from soft white winter wheat (SWWW) was challenging but can be performed (Figure S2B). SWWW seeds are high in puroindoline $A$ and large when compared to $B$. distachyon seeds and rice kernels. These seeds crumble frequently when sectioning using the telescope assemblage.

\begin{tabular}{|c|c|c|}
\hline Genotype & $\begin{array}{c}\text { Mean section width }(\boldsymbol{\mu m}) \\
\text { using telescope assemblage }\end{array}$ & $\begin{array}{c}\text { Mean section width }(\boldsymbol{\mu m}) \\
\text { sectioning freehand }\end{array}$ \\
\hline Nipponbare (husked) & $971.7 \pm 152.4^{\mathrm{ab}}$ & $1059.571 \pm 394.2^{\mathrm{ab}}$ \\
\hline Xieyou 7954 & $825.1 \pm 128.3^{\mathrm{b}}$ & $1306.187 \pm 179.1^{\mathrm{a}}$ \\
\hline RS4 & $910.6 \pm 165.0^{\mathrm{ab}}$ & $1126.694 \pm 395.3^{\mathrm{ab}}$ \\
\hline
\end{tabular}


Means followed by the same letters are not significantly different at $P<0.01$ using a one-way analysis of variance (ANOVA) and Tukey's test $(n=10)$. Statistical analyses were performed using JMP 15 software.

Table 1: Mean kernel section thickness.

\begin{tabular}{|c|c|}
\hline Genotype & Mean time (s) $^{*}$ \\
\hline Nipponbare (husked) & $14.7 \pm 1.36^{\mathrm{a}}$ \\
\hline Xieyou 7954 & $9.81 \pm 0.98^{\mathrm{b}}$ \\
\hline RS4 & $11.9 \pm 1.28^{\mathrm{c}}$ \\
\hline
\end{tabular}

*Using the telescope assemblage.

Means followed by the same letters are not significantly different at $P<0.01$ using a one-way analysis of variance (ANOVA) and Tukey's test $(n=10)$. Statistical analyses were performed using JMP 15 software.

Table 2: Mean sample preparation time.

\begin{tabular}{|c|c|c|}
\hline Genotype & Background & Quality \\
\hline Nipponbare & Wild type & Translucent \\
\hline Substandard starch grain1 (ssg1) & Nipponbare & Translucent \\
\hline Resistant Starch (RS) Xieyou 7954 & Wild type & Translucent \\
\hline RS111 & Xieyou 7954 Chalky \\
\hline RS4 & RS111 & Chalky \\
\hline Yi-Tang, 'New Life', Lujuren brand & Xieyou 7954 & Translucent \\
\hline Xiushui 11 & Wild type & Translucent \\
\hline Kemingdao1 (KMD1) & Xiushui 11 & Col \\
\hline
\end{tabular}

Table 3: Rice genotypes examined in this study. 

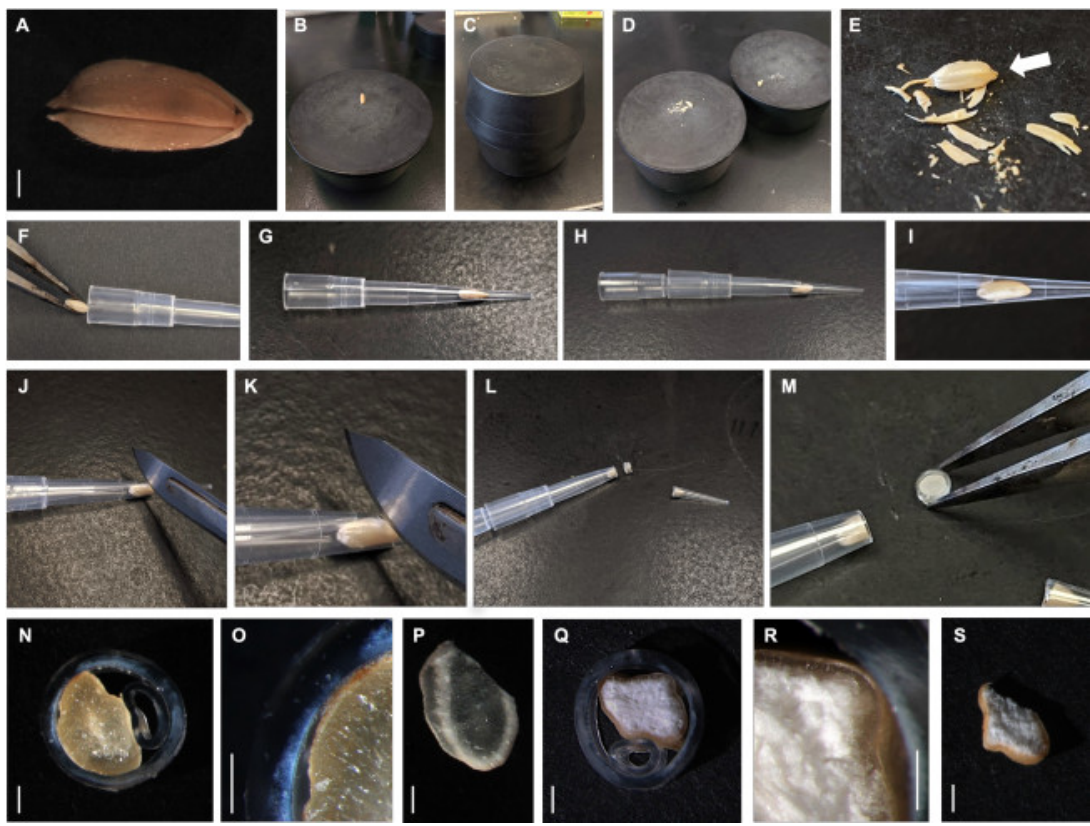

Figure 1: Preparation of transverse rice sections. (A) Wild type Nipponbare kernel with intact husk. (B). Kernel placed on a flat four-inch diameter rubber stopper. (C) Husks were removed by grinding the kernel between two apposing rubber stoppers. (D) Husk has been separated from the rice kernel. (E) Close-up of husked rice kernel. Embryo end is indicated. (F) Insertion of kernel into pipette tip using fine forceps. (G) Kernel was lodged into the distal end of the pipette tip. (H) Insertion of the second pipette tip to immobilize the kernel for sectioning (the 'telescope' assemblage). (I) The rice kernel was fitted snugly into the distal end of the pipette tip. (J) Sectioning of the rice kernel within the assemblage. (K) Close-up of the section cut. (L) A section of the kernel enclosed by the plastic annulus. (M) Close-up of the transverse section. (N) Transverse section of wild type Nipponbare. (0) Close-up of the endosperm within the wild type Nipponbare section. (P) Poor, suboptimal section of wild type Nipponbare kernel. (Q) Transverse section of Nipponbare mutant $\operatorname{ssg} 1^{4}$. (R) Closeup of the endosperm within the ssg1 section. (S) Poor, suboptimal section of ssg1. Bar (panels $\mathbf{A}, \mathbf{N}-\mathbf{S})=1 \mathrm{~mm}$. Whole rice kernel and sections were imaged using a stereomicroscope with a digital zoom camera and gooseneck lights. Please click here to view a larger version of this figure. 

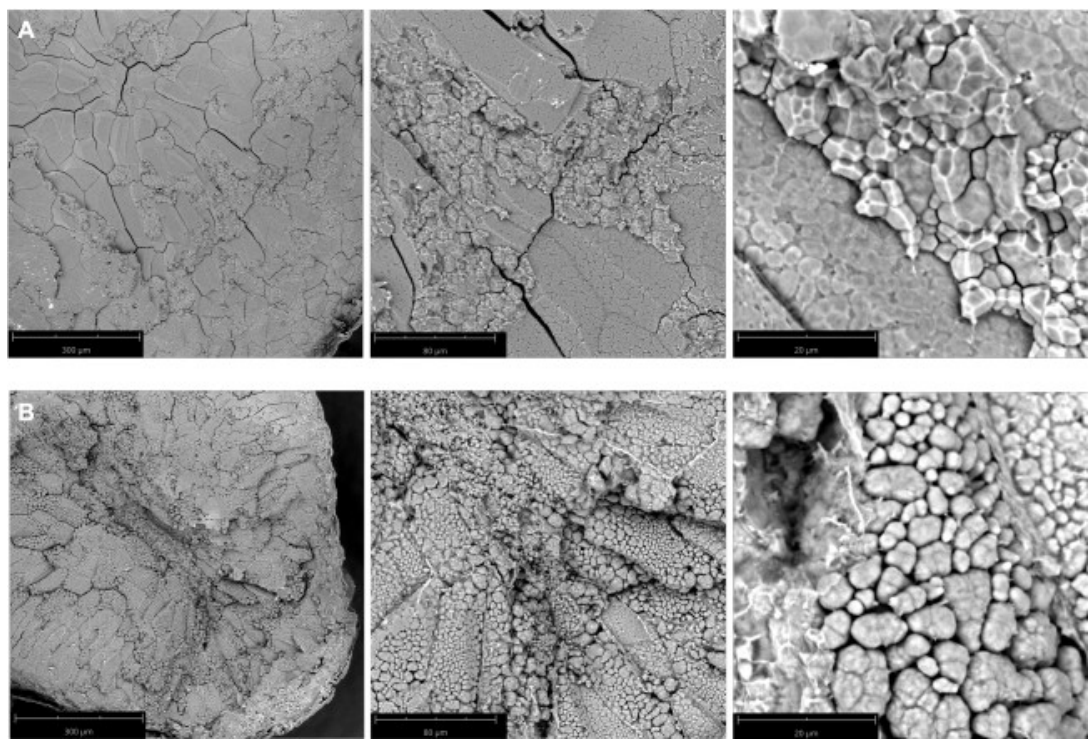

Figure 2: SEM images of transverse kernel sections. (A) Wild type Nipponbare, a translucent cultivar. The compound starch granules were cemented tightly to one another; (B) Nipponbare mutant $s s g 1^{4}$, a chalky phenotype. The compound starch granules were loosely packed and lack the cementitious nature of wild type Nipponbare starch morphotype.

Magnification from left to right: 260x, 920x, and 4200x. Bar length is indicated in panels. Please click here to view a larger version of this figure.
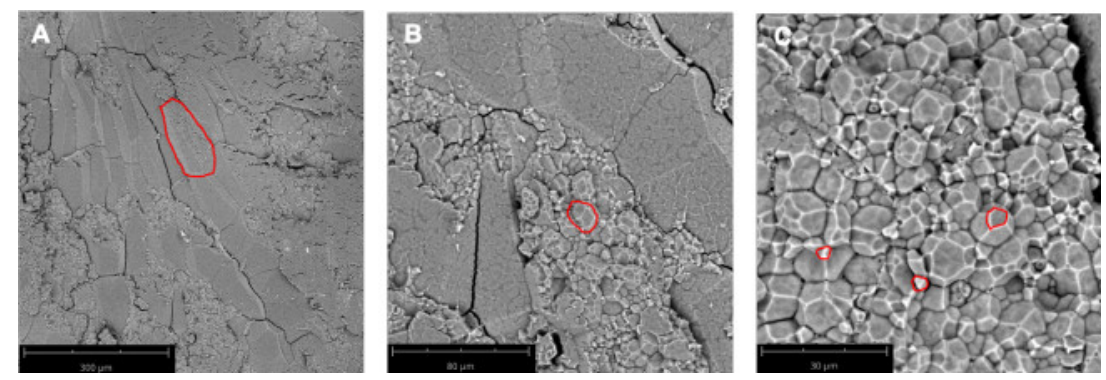

Figure 3: SEM microscopic anatomy of a transverse kernel section of Xiushui 11. (A) A single endosperm cell is outlined in red. 260x magnification. (B) A compound starch granule is outlined in red. 920x magnification. (C) Multiple starch subgranules are outlined in red. 2250x magnification. Bar lengths are indicated in the panels. Please click here to view a larger version of this figure.

Figure S1: Transverse sections of other rice genotypes prepared for SEM using this technique. (A) Resistant
Starch (RS) Xieyou $7954^{12}$.

(B) RS111, a high-RS transparent mutant of $7954^{13}$. (C) RS4, a chalky mutant of 
RS111 15 . (D) Yi-Tang, a commercial variety of high amylose rice $^{16}$. (E) Xiushui 11. (F) KMD1 (Kemingdao1) 17, 18, 19 . 10x magnification for bright field images. White bar $=1 \mathrm{~mm} .2250 \mathrm{x}$ magnification for SEM images. Bar lengths are indicated in the panels. Please click here to download this figure.

Figure S2: Technique is useful for other seeds. (A) Transverse section of false purple brome (Brachypodium distachyon L. accession Bd21) seed. (B) Transverse section of soft white winter wheat (Triticum aestivum L. cv. Augusta) seed. Bright field, 20x magnification. Bar $=1 \mathrm{~mm}$. Please click here to download this figure.

\section{Discussion}

The technique presented here represents a fast, simple, and keen approach towards preparing transverse rice cross sections for desktop SEM visualization. This sectioning technique allows for the rapid observation of endosperm structure, endosperm cell shape, size, and pattern, compound granules, and starch morphology. For the purposes of endosperm phenotyping and germplasm screening, it is critical to obtain an entire cross section of the rice kernel ${ }^{4,23,24}$. It is paramount to insert the kernel entirely within the pipette tip to prevent the pressure of the scalpel blade from forcing the endosperm to crumble or shatter. Provided the 'telescope' assemblage is properly constructed, samples can be prepared for visualization within 15 seconds (Table 2) employing materials already in hand in a typical laboratory setting. This technique is applicable to the cross-sectioning of any ellipsoidal seed approximately four millimetres in diameter at its widest point. Seeds of the model grass Brachypodium distachyon (Figure S2A) can be similarly sectioned but do not stay enclosed within the annulus. Larger seeds, like wheat, fracture easily and require care when sectioning (Figure S2B).

However, there are several limitations to the technique presented here. Sections obtained using this technique are not thin enough for the light to pass through which prohibits the use of this technique for transmitted-light based microscopic approaches like bright field (500 $\mu \mathrm{m}$ maximum sample thickness for rice kernel sections ${ }^{25}$ ) and transmission electron microscopy (TEM) (500 nm maximum sample thickness $\left.{ }^{26}\right)$. The use of a pipette tip as the sectioning 'matrix' also limits the size of seed that can be sectioned using this technique. Further troubleshooting would be required to adapt this technique for species highly dissimilar from rice, and the size of the 'matrix' is limited by the size of pipette tips available for purchase.

Another distinct advantage that this technique provides is the quality of samples that can be produced from chalky phenotype rice kernels. It is worth noting that even the Matsushima study conceded that it was difficult to obtain cross sections using that particular method for chalky phenotypes ${ }^{4}$, as replicated in this study for the purpose of comparison (Figure 1S). In their case, it became necessary to chemically fix their chalky rice samples and embed them in resin for sectioning. The new technique, in conjunction with desktop SEM imaging, allows the researcher to easily prepare transverse sections of rice kernels for microscopy with more consistency than without immobilization support (Table 3).

In the new era of phenomics and metabolomics, it is important to monitor mutagenized lines and transposon-tagged libraries to better understand the function and importance of starch in seeds. Additionally, the International Rice Genebank holds over 130000 rice accessions ${ }^{27}$. A rapid seed phenotyping technique like the one presented here would expedite 
classification and sampling for nutritional quality ${ }^{28}$. Lastly, this technique may be useful in the light of encroaching climate change impacts. Seasonal high-temperature stress during grain-filling had already been identified as a major cause of chalkiness ${ }^{6}$, but recent studies have implicated rising global temperatures in increasing chalkiness of rice yields ${ }^{7,29}$. Such expedited endosperm phenotyping may help provide a broad agricultural image of the effect of increasing global temperatures.

\section{Disclosures}

The authors have nothing to disclose.

\section{Acknowledgments}

The authors are grateful to Systems for Research (SFR Corp.) for use of their Phenom ProX Desktop SEM instrument, as well as for the technical assistance provided by Maria Pilarinos (Systems for Research (SFR) Corp.) and Chloë van Oostende-Triplet (Cell Biology and Image Acquisition Core Facility, Faculty of Medicine, University of Ottawa). Funding was provided by the Low Carbon Innovation Fund (LCIF) from the Government of Ontario's Ministry of Economic Development, Job Creation and Trade, and Proteins Easy Corp.

\section{References}

1. James, M. G., Denyer, K., Myers, A. M. Starch synthesis in the cereal endosperm. Current Opinion in Plant Biology. 6 (3), 215-222 (2003).

2. Shapter, F. M., Henry, R. J., Lee, L. S. Endosperm and starch granule morphology in wild cereal relatives. Plant Genetic Resources. 6 (2), 85-97 (2008).
3. Ashida, K., lida, S., Yasui, T. Morphological, physical, and chemical properties of grain and flour from chalky rice mutants. Cereal Chemistry. 86 (2), 225-231 (2009).

4. Matsushima, R., Maekawa, M., Fujita, N., Sakamoto, W. A rapid, direct observation method to isolate mutants with defects in starch grain morphology in rice. Plant and Cell Physiology. 51 (5), 728-741 (2010).

5. Zhao, X. et al. Identification of stable QTLs causing chalk in rice grains in nine environments. Theoretical and Applied Genetics. 129 (1), 141-153 (2016).

6. Nagato, K., Ebata, M. Effects of high temperature during ripening period on the development and the quality of rice kernels. Japanese Journal of Crop Science. 34 (1), 59-66 (1965).

7. Zhao, X., Fitzgerald, M. Climate change: implications for the yield of edible rice. PLoS One. 8 (6), e66218 (2013).

8. Zhao, Z. K., Mu, T. H., Zhang, M., Richel, A. Effects of high hydrostatic pressure and microbial transglutaminase treatment on structure and gelation properties of sweet potato protein. LWT - Food Science and Technology. 115, e108436 (2019).

9. Feiz, L. et al. Puroindolines co-localize to the starch granule surface and increase seed bound polar lipid content. Journal of Cereal Science. 50 (1), 91-98 (2009).

10. Zhao, L., Pan, T., Guo, D., Wei, C. A simple and rapid method for preparing the whole section of starchy seed to investigate the morphology and distribution of starch in different regions of seed. Plant Methods. 14 (1), 16 (2018).

11. Zhao, L., Pan, T., Cai, C., Wang, J., Wei, C. Application of whole sections of mature cereal seeds to visualize the morphology of endosperm cell and starch and the 
distribution of storage protein. Journal of Cereal Science. 71, 19-27 (2016).

12. Li, C., Dong, S., Li, G., Yuan, G., Dong, W. Breeding and application of the new combination of hybrid rice "Xieyou 7954". Journal of Zhejiang University (Agriculture and Life Sciences). 19 (3), 179-181 (2002).

13. Shu, X., Jia, L., Ye, H., Li, C., Wu, D. Slow digestion properties of rice different in resistant starch. Journal of Agricultural and Food Chemistry. 57 (16), 7552-7559 (2009).

14. Zhou, H. et al. Critical roles of soluble starch synthase SSIIla and granule-bound starch synthase Waxy in synthesizing resistant starch in rice. Proceedings of the National Academy of Sciences. 113 (45), 12844-12849 (2016).

15. Yang, C. Z. et al. Starch properties of mutant rice high in resistant starch. Journal of Agricultural and Food Chemistry. 54, 523-528 (2006).

16. Zhou, Y., Zou, Y., Jiang, Y., Li, B. Detection methods for resistance starch content of Yi-Tang rice and optimization of pretreatment. Food Science and Biotechnology. 36, 416-419 (2017).

17. Cheng, X., Sardana, R., Kaplan, H., Altosaar, I. Agrobacterium-transformed rice plants expressing synthetic $\operatorname{cry} I A(b)$ and $\operatorname{cryl} A(c)$ genes are highly toxic to striped stem borer and yellow stem borer. Proceedings of the National Academy of Sciences of the United States of America. 95 (6), 2767-2772 (1998).

18. Liu, H. et al. Rapid detection of P-35S and Tnos in genetically modified organisms by recombinase polymerase amplification combined with a lateral flow strip. Food Control. 107, e106775 (2020).
19. Shu, Q. et al. Transgenic rice plants with a synthetic cry1Ab gene from Bacillus thuringiensis were highly resistant to eight lepidopteran rice pest species. Molecular Breeding. 6 (4), 433-439 (2000).

20. Lisle, A. J., Martin, M., Fitzgerald, M. A. Chalky and translucent rice grains differ in starch composition and structure and cooking properties. Cereal Chemistry. 77 (5), 627-632 (2000).

21. Chen, G. et al. Dynamic development of starch granules and the regulation of starch biosynthesis in Brachypodium distachyon: comparison with common wheat and Aegilops peregrina. BMC Plant Biology. 14 (1), 198 (2014).

22. Giroux, M. J., Morris, C. F. Wheat grain hardness results from highly conserved mutations in the friabilin components puroindoline a and b. Proceedings of the National Academy of Sciences of the United States of America. 95 (11), 6262-6266 (1998).

23. Matsushima, R., Hisano, H. Imaging amyloplasts in the developing endosperm of barley and rice. Scientific Reports. 9, e3745 (2019).

24. Matsushima, R. et al. Amyloplast-localized SUBSTANDARD STARCH GRAIN4 protein influences the size of starch grains in rice endosperm. Plant Physiology. 164 (2), 623-636 (2014).

25. Monjardino, P. et al. Development of flange and reticulate wall ingrowths in maize (Zea mays L.) endosperm transfer cells. Protoplasma. 250 (2), 495-503 (2013).

26. Tizro, P., Choi, C., Khanlou, N. Sample preparation for transmission electron microscopy. Methods in Molecular Biology. 1897, 417-424 (2019). 
27. International Rice Genebank., www.irri.org Accessed April 16 2020. (2018).

28. Liu, Q.H., Zhou, X.B., Yang, L.Q., Li, T. Effects of chalkiness on cooking, eating and nutritional qualities of rice in two indica varieties. Rice Science. 16 (2), 161-164 (2009).

29. Morita, S., Wada, H., Matsue, Y. Countermeasures for heat damage in rice grain quality under climate change. Plant Production Science. 19 (1), 1-11 (2016). 